\title{
Simulação Computacional para Auxílio na Determinação do Escoamento da Coleta Seletiva de Resíduos
}

\author{
Eugênio de Oliveira Simonetto ${ }^{1}$, Denis Borenstein ${ }^{2}$ \\ ${ }^{1}$ Centro Federal de Educação Tecnológica de São Vicente do Sul (CEFET-SVS) \\ Vinte de Setembro, S/N - 97420-000 - São Vicente do Sul - RS - Brasil \\ ${ }^{2}$ Escola de Administração-Universidade Federal do Rio Grande do Sul (UFRGS) \\ Rua Washington Luiz, 855 - 90010-460 - Porto Alegre-RS-Brasil \\ eosimonetto@cefetsvs.gov.br , denisb@ea.ufrgs.br
}

\begin{abstract}
Resumo - O artigo apresenta a concepção, desenvolvimento e validação de um modelo de simulação de eventos discretos aplicado ao controle da distribuição da coleta seletiva de resíduos sólidos. O trabalho tem por funcionalidade principal à geração de alternativas ao processo decisório no que se refere à determinação da quantidade diária de resíduos sólidos a ser enviado a cada unidade de triagem participante do processo, de modo a evitar o desperdício de mão-de-obra, e também na busca da redução da quantidade de resíduos enviada aos aterros sanitários. Para o desenvolvimento do mesmo foi utilizado o software Arena e para a sua validação foram utilizados dados da coleta seletiva de um município do Rio Grande do Sul.
\end{abstract}

Palavras-chave: Sistemas de Apoio à Decisão, Gestão de Resíduos Sólidos, Simulação Computacional

\section{Introdução}

A reciclagem dos resíduos sólidos é uma excelente alternativa para propiciar a preservação de recursos naturais, a economia de energia, redução de área que demanda o aterro sanitário, geração de emprego e renda, assim como a conscientização da população para questões ambientais. Porém, para um melhor funcionamento, é de vital importância que se implante nas cidades um amplo sistema de coleta seletiva, onde os recicláveis sejam separados nas residências e coletados pelo sistema municipal de coleta seletiva. Apesar de ser uma excelente alternativa para a redução de resíduos com destino aos aterros, apenas 4,7\% dos resíduos são reutilizados ou reciclados nas cidades gaúchas, segundo a CEMPRE (Organização Não-Governamental Compromisso Empresarial para Reciclagem) (NETTO, 2001). Um dos motivos para tal parcela reduzida de reciclagem deve-se ao mau acondicionamento dos resíduos pela população, fato este gerado pela falta de informação acerca da coleta seletiva. Outros fatores que contribuem para o pequeno índice de reciclagem dos resíduos é o alto custo da coleta seletiva para as municipalidades (O'LEARY et al., 1999; MONTEIRO et al., 2001), assim como a falta de um sistema corretamente dimensionado em termos da capacidade de armazenamento e processamento de resíduos nas unidades de triagem.

Além do alto custo da coleta, um fator que contribui bastante para os baixos índices de reciclagem é a falta de atenção dada à capacidade de armazenamento e processamento de 
resíduos nas unidades de triagem. Muitas vezes, modelos desenvolvidos para o projeto e operação de sistemas de coleta consideram, para a definição da distribuição dos resíduos, somente as distâncias ou os custos de deslocamentos dos pontos de coleta aos depósitos finais (CHANG \& WEI, 2000; HUANG et al., 1998; TUNG \& PINNOI, 2000). Tais modelos não levam em consideração o fluxo dinâmico de resíduos (entrada / saída) que possuem as unidades de triagem de resíduos potencialmente recicláveis. Particularmente, Huang et al. (1998) trata o armazenamento de recicláveis, mas não dedica uma atenção especial ao fluxo de entrada e saída dos mesmos nas unidades de triagem, pois atribui à variável fluxo um valor médio calculado a partir de uma faixa de valores pré-determinada. Contudo, no caso da distribuição da coleta seletiva de resíduos, não se pode tratar somente a minimização do custo de deslocamentos ou a distância percorrida pelos veículos coletores, tem-se de levar em consideração a capacidade física e de trabalho diária das unidades de triagem de resíduos, pois muitas vezes pode existir uma solução com um custo menor do que outra, mas que a unidade de triagem em determinado momento não suporte $o$ carregamento a ela destinado. Neste caso, quase sempre estes resíduos são destinados diretamente ao aterro sanitário, deixando de serem reaproveitados, e, portanto, não gerando renda para os trabalhadores da unidade. Ao mesmo tempo uma outra unidade de triagem, por ser menos vantajosa em termos de custo ou distância, pode não ter matéria-prima a ser processada, resultando em menor quantidade de lixo reaproveitada, mão-de-obra ociosa e sobrecarga desnecessária ao aterro sanitário. Tais características, não levadas em conta na literatura especializada, são devidamente tratadas neste trabalho.

Para o desenvolvimento do sistema computacional foram utilizadas técnicas quantitativas oriundas da Pesquisa Operacional, no caso, a simulação discreta. O uso desta técnica objetiva agregar qualidade ao processo decisório, pois em muitas vezes as decisões sobre o planejamento da gestão dos resíduos sólidos são tomadas baseadas somente na experiência dos gestores (CHANG \& WEI, 2000). Tal fato, segundo este autor, contribui para o alto custo e o baixo desempenho dos sistemas de coleta de resíduos nas cidades. A utilização de ferramentas de Pesquisa Operacional (PO) na Gestão de Resíduos Sólidos surge como uma alternativa viável para o tratamento da complexidade inerente ao processo de coleta seletiva de resíduos sólidos, pois através do uso destas ferramentas pode-se representar uma situação do mundo real, estudar seu comportamento (via execução de modelos formais) e tomar decisões com base nas conclusões extraídas. Vários autores (HUANG et al., 1998; CHANG \& WEI, 2000; BHAT, 1996; EVERETT \& SHAHI, 1996; KULCAR, 1996; TUNG \& PINNOI, 2000;) já utilizaram técnicas e métodos da PO para desenvolver estudos na área de coleta de resíduos sólidos.

Com o desenvolvimento do modelo de simulação, pretende-se auxiliar no planejamento da distribuição da coleta seletiva de resíduos sólidos, buscando, reduzir a quantidade de resíduos recicláveis desperdiçados devido à falta de controle na capacidade de armazenagem e processamento de trabalho nas unidades de triagem deste tipo de resíduo.

O artigo está organizado da seguinte forma: na seção 2 é apresentada a coleta seletiva de resíduos sólidos, na seção 3 são apresentados os conceitos relativos à simulação computacional. Na seção 4 é descrito o modelo de simulação desenvolvido, bem como na seção 5 é apresentada a validação do mesmo. Por fim, na seção 6 são apresentadas as considerações finais do artigo. 


\section{A Coleta Seletiva de Resíduos}

A coleta seletiva é o modelo mais empregado nos programas de reciclagem e consiste na separação, pela população, dos materiais recicláveis existentes nos resíduos domésticos para que posteriormente os mesmos sejam coletados por um veículo específico (BARLAZ et al., 1995). A separação dos materiais recicláveis nas residências pode ser feita individualizando-se os materiais recicláveis e acondicionando-os em contêineres diferenciados ou agrupando-os em um único recipiente (CHANG \& WEI, 2000). O fundamento deste processo é a separação, pela população, dos materiais recicláveis (compostos inorgânicos) do restante do lixo (compostos orgânicos), que é destinado a aterros ou usinas de compostagem.

Após a coleta, os materiais recicláveis devem ser transportados para uma unidade de triagem, equipada com lugares para catação, para que seja feita uma separação mais criteriosa dos materiais visando a comercialização dos mesmos. As unidades de triagem devem ser dotadas de prensas para que os materiais recicláveis de menor peso específico (papéis e plásticos) possam ser enfardados para facilitar a estocagem e o transporte dos mesmos. É importante que a população seja devidamente orientada para que somente sejam separados, como lixo seco, os materiais que possam ser comercializados, evitando-se despesas adicionais com o transporte e manuseio de rejeitos, que certamente serão produzidos durante o processo de seleção por tipo de material e no enfardamento.

A distribuição dos resíduos entre as unidades se dá de acordo com a capacidade de recepção de cada unidade, em função das dimensões da mesma, do número de pessoas envolvidas e do ritmo de trabalho das pessoas envolvidas. O modelo de simulação busca estimar a quantidade de resíduos a ser enviada a cada unidade de triagem tendo por base os fatores apresentados anteriormente.

\section{A Simulação Computacional}

A simulação computacional de sistemas consiste na utilização de um conjunto de métodos e técnicas matemáticas, com o objetivo de imitar o comportamento de sistemas reais, geralmente utilizando-se computadores e softwares para tal (KELTON et al., 1998). Ela pode ser definida como um processo de projetar um modelo de um sistema real e, de procedimentos de experimentos com este modelo, com o propósito de verificar o comportamento do sistema, ou até mesmo avaliar estratégias para a sua operação (PEGDEN et al., 1995).

Através do uso da simulação, pode-se imitar o comportamento de, praticamente, qualquer tipo de operação ou processo do mundo real (LAW \& KELTON, 1991). Uma das principais etapas de um estudo de simulação consiste na criação de um modelo lógico. Assim, um modelo lógico consiste em um conjunto de suposições e aproximações, devidamente quantificadas e estruturadas, que visam representar o comportamento do sistema real sob determinadas condições, utilizando-o para predizer e comparar alternativas lógicas passíveis de serem simuladas. 
Um modelo é utilizado também quando se deseja aprender alguma coisa sobre o sistema real que não se pode observar ou experimentar diretamente, ou pela inexistência do sistema real, ou pela dificuldade de manipulação do mesmo, ou pela impossibilidade de sujeitá-lo ao ensaio sem incorrer em custos elevados e gastos de tempo. A quantidade de simplificações impostas ao modelo influenciará em razão direta na distorção entre os resultados obtidos pelo modelo e pelo sistema real.

A simulação permite também a utilização de variáveis aleatórias, de modo a representar com maior veracidade o comportamento do sistema real. Portanto, este método se constitui numa das ferramentas de análise mais poderosas disponíveis para a representação de sistemas complexos (KELTON et al., 1998). A simulação possui várias aplicações no cotidiano, nas áreas mais diversas, que vão desde o controle de produção em na área de alimentos, do controle do fluxo de papéis em um escritório, do controle do trânsito nas cidades até ao controle do transporte de instrumentos cirúrgicos dentro de um hospital.

\section{A Simulação do Processamento de Resíduos nas Unidades de Triagem}

O modelo de simulação desenvolvido tem como principal finalidade estimar a capacidade de processamento de resíduos sólidos nas unidades de triagem consideradas no processo decisório acerca da coleta seletiva. A determinação da capacidade de processamento é uma particularidade do caso de resíduos potencialmente recicláveis e, é originado pelo fluxo de entrada e saída deste tipo de resíduo nas unidades de triagem. Fato este, não verificado no caso dos resíduos sólidos que são direcionados ao aterro sanitário, já que não existe a saída dos resíduos sólidos (somente entrada de resíduos) neste tipo de disposição final (CHANG \& WEI, 2000; HUANG et al., 1998). Para a simulação, utiliza-se o software comercial ARENA (ARENA SOFTWARE, 2004).

Para auxílio na determinação da produtividade das unidades de triagem (quantidade de resíduos processados diariamente por pessoa) foi utilizado o modelo proposto por Lajolo (2003). No referido modelo, basicamente, são levados em consideração o total de resíduos processados diariamente e o número de horas trabalhadas diárias para obtenção da produtividade. Um fato que merece destaque é a variação na produtividade de um separador para outro, pois foram verificados casos, em uma mesma unidade de triagem, que um separador processou $330 \mathrm{~kg} / \mathrm{dia}$, enquanto outro processou somente $125 \mathrm{~kg}$ (LAJOLO, 2003).

O modelo de simulação é composto, basicamente, pelas seguintes variáveis: quantidade de resíduos potencialmente recicláveis a serem coletados, quantidade de resíduos armazenados por unidade de triagem e quantidade de resíduos processados por unidade de triagem. As variáveis aleatórias componentes do modelo de simulação são: taxa média de geração de resíduo por pessoa em área de coleta, taxa média de chegada de resíduos na unidade de triagem e a taxa média de processamento de resíduo em cada unidade.

Quando da utilização do modelo de simulação o usuário irá informar, em interface desenvolvida para tal funcionalidade, o dia da semana para o qual será efetuado o planejamento da coleta seletiva, o mês do ano (de forma a considerar as eventuais sazonalidades existentes no processo), as unidades de triagem de resíduos operantes, para que seja determinada a quantidade máxima de resíduos sólidos (em $\mathrm{kg}$ ), que cada unidade em operação é capaz de processar diariamente. A interface para o usuário informar os dados citados anteriormente é apresentada na figura 1. Já a interface da simulação no Arena em execução é apresentada na figura 2. 
Para a execução da simulação, a qual determinará a capacidade de processamento de resíduos de cada unidade de triagem, são necessárias as seguintes informações:

- taxa média de geração de resíduos - quantidade média de resíduos gerados pela população. A taxa é expressa em quilogramas por minuto.

- quantidade de resíduos em espera por processamento - quantidade de resíduos aguardando por processamento (triagem) em cada unidade de triagem. Esta informação é expressa em quilogramas;

- ritmos médios de processamento de resíduos pelos selecionadores - quantidade média de resíduos que cada selecionador é capaz de processar. O ritmo médio dos selecionadores é expresso em quilogramas por minuto.

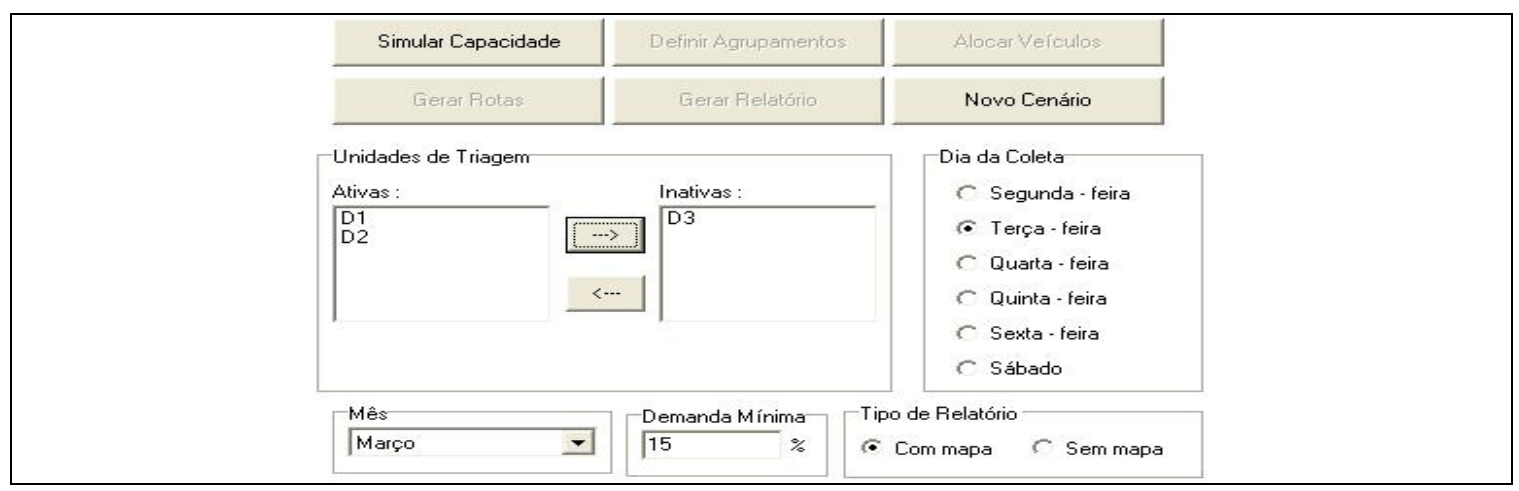

Figura 1 - Interface para o usuário informar dados relativos à simulação

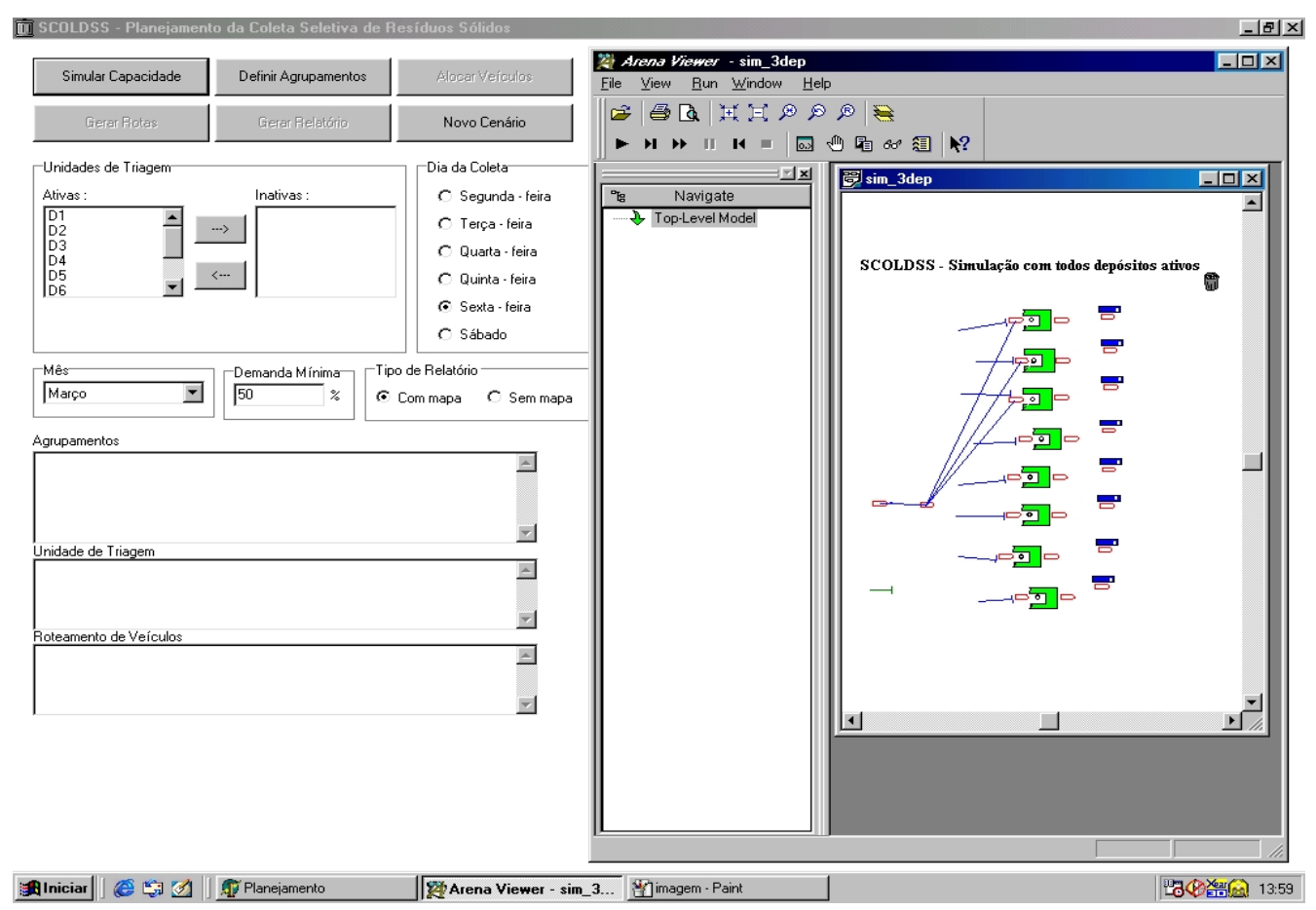

Figura 2 - Interface referente à execução do modelo de simulação

A identificação das informações anteriormente descritas influenciará na determinação da quantidade total de resíduos processados por dia nas unidades de triagem. A quantidade 
total de resíduos processados, tanto os resíduos selecionados para retornarem ao mercado, como os resíduos que terão como destino o aterro sanitário, têm de serem levados em consideração pela simulação, pois ambos ocupam espaço físico temporariamente e também consumiram um determinado tempo para serem processados pelos trabalhadores.

Para a distribuição dos resíduos sólidos entre as unidades, durante a simulação, foi utilizado o componente Pickstation do simulador Arena, o qual seleciona a unidade para enviar a matéria-prima (resíduo), em ordem de precedência, de acordo com o número de recursos (pessoas) utilizados (de modo a evitar ociosidade) e, pela quantidade de matériaprima pós-consumo aguardando para ser processada, pois em cada unidade de triagem é definida uma capacidade máxima de armazenamento de resíduos. Nesta seleção, no modelo de simulação, podem ser consideradas eventuais interrupções no trabalho (almoço, troca de equipe de trabalho) e a variação da produção de um turno para outro.

Para ilustrar o desenvolvimento do processo de modelagem da simulação discreta foi utilizado o Diagrama de Ciclo de Atividade, pois segundo Pidd (1998), a diagramação auxilia a compreensão do modelo e suas interações. No diagrama de ciclo de atividades os estados ativos são representados por retângulos e têm sua duração estabelecida por eventos, cuja duração é determinada a partir de uma distribuição probabilística. Já os estados inativos, também chamados de estado de espera, são representados por círculos e ocorrem quando uma entidade está esperando que algo aconteça no sistema, por isso são conhecidos também por "fila”. O diagrama de ciclo de atividade do modelo de simulação é apresentado na figura 3.

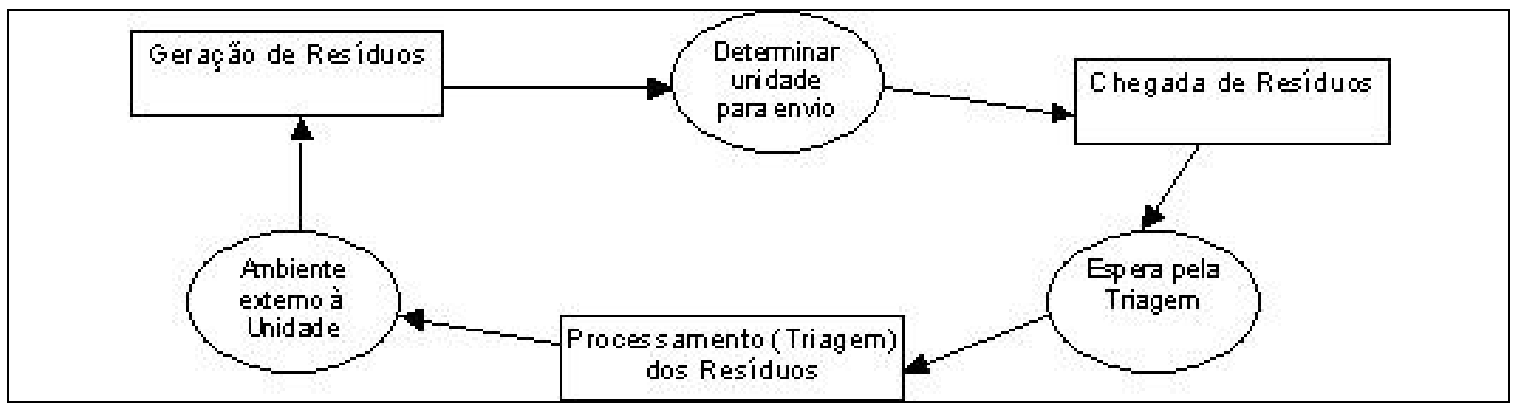

Figura 3 - Diagrama de Ciclo de Atividade do Modelo de Simulação

A informação gerada é a demanda de resíduos sólidos (em $\mathrm{kg}$ ) que cada unidade de triagem é capaz de processar em um determinado dia de trabalho, obtida pela média das $n$ rodadas da simulação

\section{Validação do Modelo de Simulação}

Para a validação do módulo de simulação da capacidade de processamento de resíduos diário das unidades de triagem foram utilizados dados reais de sete das oito unidades e, foram coletados dados referentes a dois meses de processamento. Nesta validação o sistema comportou-se de maneira correta, com um desvio médio de 1,01\% dos dados reais coletados, o que não compromete o desempenho do sistema, tendo em vista que a simulação trata da capacidade máxima de processamento. Na validação do módulo de simulação da capacidade de processamento das unidades de triagem foram utilizadas as técnicas quantitativa e qualitativa proposta por O’Keefe (1987), a técnica quantitativa no que se refere à comparação dos resultados com os dados reais das unidades de triagem e, a abordagem qualitativa no que refere-se a construção do modelo de simulação, ou seja, na verificação se o modelo retrata de uma forma aceitável o processo existente na realidade. 
As execuções da simulação do processamento de resíduos foram desenvolvidas da seguinte forma: para cada dia da semana executou-se vinte rodadas do modelo de simulação e, que o tempo médio destas execuções foi de 13 minutos e 16 segundos. O desvio médio dos resultados obtidos, em relação aos dados reais (atuais) de processamento nas unidades foi de $1,01 \%$. Tal desvio é considerado insignificante tendo em vista que são simuladas capacidades máximas de processamento de resíduos. As simulações foram desenvolvidas utilizando um computador Pentium II de $1.6 \mathrm{GHz}$ e $256 \mathrm{Mb}$ de memória RAM. Os resultados referentes à simulação do processamento de resíduos nas unidades de triagem são apresentados na tabela 1 e, correspondem ao cálculo médio referente a cem rodadas da simulação. Os valores expressam o total em quilogramas que cada unidade pode processar no dia especificado.

\begin{tabular}{|c|c|c|c|c|c|}
\hline & Segunda & Terça & Quarta & Quinta & Sexta \\
\hline $\begin{array}{c}\text { Unidade de } \\
\text { Triagem 1 }\end{array}$ & $10600 \mathrm{~kg}$ & $10700 \mathrm{~kg}$ & $10600 \mathrm{~kg}$ & $10600 \mathrm{~kg}$ & $10550 \mathrm{~kg}$ \\
\hline $\begin{array}{c}\text { Unidade de } \\
\text { Triagem 2 }\end{array}$ & $7200 \mathrm{~kg}$ & $7200 \mathrm{~kg}$ & $7320 \mathrm{~kg}$ & $7280 \mathrm{~kg}$ & $7300 \mathrm{~kg}$ \\
\hline $\begin{array}{c}\text { Unidade de } \\
\text { Triagem 3 }\end{array}$ & $7220 \mathrm{~kg}$ & $7240 \mathrm{~kg}$ & $7200 \mathrm{~kg}$ & $7240 \mathrm{~kg}$ & $7210 \mathrm{~kg}$ \\
\hline $\begin{array}{c}\text { Unidade de } \\
\text { Triagem 4 }\end{array}$ & $5900 \mathrm{~kg}$ & $5850 \mathrm{~kg}$ & $5800 \mathrm{~kg}$ & $5850 \mathrm{~kg}$ & $5900 \mathrm{~kg}$ \\
\hline $\begin{array}{c}\text { Unidade de } \\
\text { Triagem 5 }\end{array}$ & $7600 \mathrm{~kg}$ & $7550 \mathrm{~kg}$ & $7600 \mathrm{~kg}$ & $7500 \mathrm{~kg}$ & $7600 \mathrm{~kg}$ \\
\hline $\begin{array}{c}\text { Unidade de } \\
\text { Triagem 6 }\end{array}$ & $6500 \mathrm{~kg}$ & $6450 \mathrm{~kg}$ & $6450 \mathrm{~kg}$ & $6500 \mathrm{~kg}$ & $6500 \mathrm{~kg}$ \\
\hline $\begin{array}{c}\text { Unidade de } \\
\text { Triagem 7 }\end{array}$ & $6400 \mathrm{~kg}$ & $6400 \mathrm{~kg}$ & $6480 \mathrm{~kg}$ & $6350 \mathrm{~kg}$ & $6370 \mathrm{~kg}$ \\
\hline $\begin{array}{c}\text { Unidade de } \\
\text { Triagem 8 }\end{array}$ & $10800 \mathrm{~kg}$ & $10800 \mathrm{~kg}$ & $10820 \mathrm{~kg}$ & $10900 \mathrm{~kg}$ & $10850 \mathrm{~kg}$ \\
\hline
\end{tabular}

Tabela 1 - Resultados das execuções da simulação do processamento de resíduos

\section{Considerações Finais}

O objetivo principal do trabalho foi o de apresentar um modelo de simulação o qual permite o auxílio aos gestores da coleta seletiva de resíduos sólidos, no que refere-se ao processo decisório acerca da quantidade de resíduo a ser destinada a cada unidade de triagem operante nos dias de coleta.

A conclusão que pode ser extraída, a partir da utilização do modelo de simulação nas unidades de triagem, é de que o modelo comportou-se de forma correta, apresentando um desvio médio de $1,01 \%$ da capacidade informada pela empresa. Tal desvio, não compromete o desempenho do sistema, pois as mesmas tratam da capacidade máxima do processamento diário de resíduos sólidos.

Um fator que merece destaque é o que diz respeito à distribuição do material coletado entre as unidades de triagem operantes nos dias de coleta, pois constatou-se que em alguns dias determinadas unidades de triagem possuíam capacidade de processamento, entretanto não recebiam nenhum material para trabalho. Com o uso do modelo de simulação tal problema pode ser facilmente solucionado, pois todo o planejamento será executado 
antecipadamente, assim consegue-se, após a execução da simulação, determinar a quantidade de resíduo que cada unidade de triagem irá receber em um dia de coleta.

\section{Referências}

ARENA SOFTWARE. Arena - Overview. Disponível na Web em: http://www.arenasoftware. com. Acessado em 16 de dezembro de 2004.

BARLAZ, M.A.; et al. Life-Cycle Study of Municipal Solid Waste Management - System Description. Washington DC: U.S. Environmental Protection Agency, 1995.

BHAT, V.N. A model for the optimal allocation of trucks for the solid waste management. Waste Management \& Research, 14, pp.87-96, 1996.

CHANG, N.; WEI, Y. Siting recycling drop-off in urban area by genetic algorithm-based fuzzy multiobjective nonlinear integer programming modeling. Fuzzy Sets and Systems, 114, pp.133149, 2000.

EVERETT, J.W.; SHAHI, S. Vehicle and labor requirements for yard waste collection. Waste Management \& Research, 15, pp.627-640, 1997.

HUANG, G.H.; BAETZ, B.W.; PATRY, G.G. Trash-Flow Allocation: Planning Under Uncertainty. Interfaces, Vol. 28, No. 6, pp. 36-55, Nov-Dec, 1998.

KELTON, W. D., SADOWSKI, R. P., SADOWSKI, D. A. Simulation with Arena, McGraw-Hill, New York, 1998.

KULCAR, T. Optimizing solid waste collection in Brussels. European Journal of Operations Research, 90, pp.71-77, 1996.

LAJOLO, R.D. Cooperativa de catadores de materiais recicláveis: Guia para implantação. São Paulo: Instituto de Pesquisas Tecnológicas-Sebrae, 2003.

LAW, A.M., KELTON, W.D. Simulation Modeling \& Analysis. $2^{\text {a }}$ Ed., McGraw-Hill, 1991.

MONTEIRO, J.H.P.; et al. Manual de Gerenciamento Integrado de Resíduos Sólidos. Rio de Janeiro: Instituto Brasileiro de Administração Municipal, 2001.

NETTO, A. O Desperdício do Lixo. Jornal Zero Hora, 09 de setembro de 2001, Página 32.

O'KEEFE, R. M.; BALCI, O.; SMITH; E.P. Validating expert system performance. IEEE Expert 2(4):81-90, 1987.

O'LEARY, P.R; et al.. Decision Maker's Guide to Solid Waste Management.Vol. 2. Washington DC: U.S. Environmental Protection Agency, 1999.

PEDGEN, C. D., SHANON, R. E., SADOWSKI, R. P. Introduction to simulation using SIMAN, McGraw-Hill, 2o. ed., 1995.

PIDD, M. Modelagem Empresarial - Ferramentas para a Tomada de Decisão. Porto Alegre: Artmed, 1998.

TUNG, D.V.; PINNOI, A. Vehicle routing-scheduling for waste collection in Hanoi. European Journal of Operational Research, 125, pp.449-468, 2000. 